\title{
Síndrome metabólico y aterosclerosis carotídea subclínica en niños y adolescentes mexicanos con acantosis nigricans
}

\author{
Cynthia Guadalupe González-Villalobos, ${ }^{1}$ Elizabeth Guevara-Gutiérrez, ${ }^{1}$ Pedro Gutiérrez-Fajardo, ${ }^{2}$ \\ José Alberto Tlacuilo-Parra, ${ }^{3}$ María Elena Sánchez-Castellanos, ${ }^{1}$ Alejandro García-Vargas ${ }^{1}$ y \\ José Fernando Barba-Gómez ${ }^{1}$ \\ ${ }^{1}$ Secretaría de Salud, Instituto Dermatológico de Jalisco "Dr. José Barba Rubio"; ${ }^{2}$ Hospital Bernardette, Laboratorio de Ecocardiografía; ${ }^{3}$ Instituto \\ Mexicano del Seguro Social, Centro Médico Nacional de Occidente, Hospital de Pediatría. Jalisco, México
}

\section{Resumen}

Introducción: La acantosis nigricans es un marcador de resistencia a la insulina, la cual se asocia con alteraciones metabólicas y cardiovasculares. Objetivo: Investigar la frecuencia de síndrome metabólico y aterosclerosis carotídea subclínica en niños y adolescentes mexicanos con acantosis nigricans y comparar los resultados entre sexos. Método: Estudio transversal. Se incluyeron 30 sujetos masculinos y 30 femeninos con diagnóstico de acantosis nigricans, menores de 18 años. Se investigó síndrome metabólico (criterios de Cook), riesgo cardiovascular (proteína C reactiva ultrasensible [PCRus]) y aterosclerosis carotídea (grosor íntima-media). Para el análisis de datos se utilizó estadística descriptiva e inferencial. Resultados: La frecuencia de síndrome metabólico fue de $43 \%$ (sexo masculino $42 \%$ versus femenino $58 \%, p=0.58$ ). Todos presentaron niveles anormales de PCRus: $67 \%$ fue clasificado con riesgo cardiovascular moderado y $27 \%$ con riesgo alto. Frecuencia de aterosclerosis carotídea $98 \%$ (masculino $49 \%$ versus femenino $51 \%, p=0.45$ ). La severidad de la acantosis nigricans no influyó en los resultados. Conclusiones: La búsqueda intencionada de síndrome metabólico y aterosclerosis carotídea subclínica en niños y adolescentes mexicanos con acantosis nigricans, independientemente del sexo o severidad de la enfermedad, permitirá implementar medidas para disminuir la morbimortalidad en la edad adulta.

PALABRAS CLAVE: Acantosis nigricans. Síndrome metabólico. Aterosclerosis carotídea. Niños. Adolescentes.

\begin{abstract}
Introduction: Acanthosis nigricans is a marker of insulin resistance that is associated with metabolic and cardiovascular alterations. Objective: To investigate the frequency of metabolic syndrome and subclinical carotid atherosclerosis in children and adolescents with acanthosis nigricans, and to compare the results between genders. Method: Cross-sectional study, where 30 male and 30 female subjects younger than 18 years of age diagnosed with acanthosis nigricans were included. The presence of metabolic syndrome (Cook's criteria), cardiovascular risk (ultrasensitive C-reactive protein and [us-CRP]), and carotid atherosclerosis (intima-media thickness [IMT]) was investigated. Descriptive and inferential statistics was used for data analysis. Results: The frequency of metabolic syndrome was $43 \%$ (males $42 \%$ versus females $58 \%, p=0.58$ ). All patients showed us-CRP abnormal levels: $67 \%$ were classified with moderate cardiovascular risk and $27 \%$ with high risk. The prevalence of carotid atherosclerosis was $98 \%$ (males $49 \%$ versus females $51 \%, p=0.45$ ). Acanthosis nigricans severity did not influence on the results. Conclusions: Intentional search for metabolic syndrome and subclinical carotid atherosclerosis in Mexican children and adolescents with acanthosis nigricans, regardless of gender or disease severity, will enable the implementation of measures to decrease the morbidity and mortality seen in adult age.
\end{abstract}

KEY WORDS: Acanthosis nigricans. Metabolic syndrome. Carotid atherosclerosis. Children. Adolescents.

Correspondencia:

José Alberto Tlacuilo-Parra

E-mail: albtlacuilo@yahoo.com
Fecha de recepción: 29-08-2017

Fecha de aceptación: 8-01-2018

DOI://dx.doi.org/10.24875/GMM.18003699
Gac Med Mex. 2018;154:462-467

Disponible en PubMed

www.gacetamedicademexico.com 


\section{Introducción}

La acantosis nigricans (AN) se considera una manifestación cutánea de la obesidad y de resistencia a la insulina. ${ }^{1}$ Esta última es la vía patogénica común entre la AN, el síndrome metabólico (SM) y la aterosclerosis, ${ }^{2}$ dado que los niveles elevados de insulina en suero tienen los siguientes efectos:

- Estimulan los receptores del factor 1 de crecimiento de insulina en los queratinocitos y fibroblastos dérmicos, lo que favorece el desarrollo de AN. ${ }^{3}$

- Producen lipólisis inapropiada con aumento de los triglicéridos y disminución de las lipoproteínas de alta densidad (HDL), que facilitan el desarrollo de SM. ${ }^{4}$

- Contribuyen al desarrollo progresivo de aterogénesis y permiten la liberación de citocinas proinflamatorias que perpetúan la resistencia a la insulina y derivan en mayor disfunción endotelial. ${ }^{5}$

Debido a que en la población pediátrica la AN ha aumentado en forma paralela al aumento de la obe$\operatorname{sidad}^{3}$ y que en México, de acuerdo con la Organización para la Cooperación y el Desarrollo Económico, se estima que $29 \%$ de las niñas y $28 \%$ de los niños presentan sobrepeso u obesidad, ${ }^{6}$ esperaríamos mayor presencia de alteraciones metabólicas y cardiovasculares en este grupo de edad, si bien en una búsqueda en la literatura no encontramos datos al respecto en población pediátrica mexicana con AN. Por ello, nuestro objetivo fue investigar la frecuencia de SM y de aterosclerosis carotídea en niños y adolescentes mexicanos con AN y comparar los resultados entre ambos sexos y con los publicados en la literatura.

\section{Método}

Estudio transversal en el Instituto Dermatológico de Jalisco "Dr. José Barba Rubio", previa autorización del Comité de Ética de la institución y firma del consentimiento previa información del padre o tutor de cada participante. Se incluyó una muestra por conveniencia de 60 pacientes con diagnóstico de AN (30 masculinos y 30 femeninos), mexicanos de nacimiento y dos generaciones previas de origen mexicano, de 0 a 17 años, sin tratamiento previo de la AN ni otras dermatosis asociadas con obesidad, diabetes o trastornos de lípidos.
Se excluyeron los sujetos que al interrogatorio refirieron el diagnóstico de enfermedad congénita o adquirida durante la evolución de la AN, alteraciones metabólicas, lipídicas o aterosclerosis y aquellos con consumo de medicamentos para disminuir de peso, hipoglicemiantes, antihipertensivos, hipolipemiantes, hormona del crecimiento, hormonas sexuales o glucocorticoides, que se asocian con el desarrollo de AN. Se eliminaron pacientes con valores de proteína $C$ reactiva ultrasensible (PCRus) $>10 \mathrm{mg} / \mathrm{L}$, por la asociación con procesos agudos inflamatorios. ${ }^{7}$

El diagnóstico de AN fue clínico y realizado por un dermatólogo capacitado. Se consideró como condición para su diagnóstico que el cuello estuviera afectado y que la severidad tuviera una puntuación $\geq 1$. Para determinar la severidad se tomaron en cuenta cinco sitios anatómicos: cuello, axilas, codos, nudillos y rodillas. El cuello se valoró respecto a la extensión (escala de 0 a 4) y la severidad (textura con una escala de 0 a 3). En la axila se valoró solo la extensión (escala del 0 al 4). En los codos, nudillos y rodillas se determinó solo presencia (1 punto) o ausencia (0 puntos). Para definir los grados de severidad de la AN, la puntuación total de evaluación fue clasificada en ausente $(<1)$, leve (1-1.5), moderada (1.6-1.9) y severa $(\geq 2){ }^{8}$

El diagnóstico de síndrome metabólico se realizó con base en los criterios de Cook, ${ }^{9}$ con la presencia de tres o más de los siguientes parámetros:

- Circunferencia de cintura $\geq$ percentil 90 para edad y sexo. Con una cinta métrica de material no distensible y el paciente en bipedestación, se midió el punto medio entre la décima segunda costilla y el reborde de la cresta ilíaca, pasando por el ombligo, después de una exhalación normal. ${ }^{10}$

- Hipertensión arterial $\geq$ percentil 90 para edad, sexo y talla. ${ }^{9}$ Se tomó en sedentación, con un esfigmomanómetro de mercurio con manguito apropiado para la longitud del brazo y la edad del paciente, después de un periodo de reposo de 10 minutos. La presión arterial se clasificó de acuerdo con los valores normales para edad, sexo y estatura, utilizando la metodología estandarizada por el Second Task Force. ${ }^{11}$

- Triglicéridos $\geq 110 \mathrm{mg} / \mathrm{dL} .{ }^{9}$ Se determinaron por método enzimático colorimétrico. Para su medición se obtuvo una muestra de sangre venosa periférica de $10 \mathrm{~mL}$ en ayuno de 12 horas, la cual sirvió también para la realización del resto de los exámenes de laboratorio. 
Tabla 1. Distribución de las variables de estudio por severidad de la acantosis nigricans

\begin{tabular}{|c|c|c|c|c|c|c|c|}
\hline \multirow[t]{2}{*}{ Componente } & \multicolumn{2}{|c|}{ AN leve $(n=16)$} & \multicolumn{2}{|c|}{ AN moderada $(n=13)$} & \multicolumn{2}{|c|}{ AN severa $(n=31)$} & \multirow[t]{2}{*}{$\mathbf{p}^{*}$} \\
\hline & $\mathrm{n}$ & $\%$ & $\mathbf{n}$ & $\%$ & $\mathbf{n}$ & $\%$ & \\
\hline Síndrome metabólico ( $\mathrm{n}=26)$ & 7 & 27 & 7 & 27 & 12 & 46 & $0.73^{\star *}$ \\
\hline Riesgo cardiovascular moderado $(n=40)$ & 11 & 27.5 & 9 & 22.5 & 20 & 50 & $0.77^{\star *}$ \\
\hline Riesgo cardiovascular alto $(n=16)$ & 4 & 25 & 3 & 19 & 9 & 56 & $0.52^{\star \star *}$ \\
\hline Aterosclerosis carotídea $(n=59)$ & 16 & 27 & 13 & 22 & 30 & 51 & $0.65^{\star * *}$ \\
\hline
\end{tabular}

*El valor de P es el resultado de la comparación entre AN leve versus severa. **Prueba de chi cuadrado. ***Prueba exacta de Fisher.

AN $=$ Acantosis nigricans.

- Colesterol HDL $\leq 40 \mathrm{mg} / \mathrm{dL}^{9}$ y glucosa $\geq 110 \mathrm{mg} / \mathrm{dL} .{ }^{9}$ Ambos se determinaron por método enzimático colorimétrico.

La aterosclerosis se determinó con el grosor íntima-media (GIM) carotídeo, definido como el tamaño de la pared distal de la arteria carótida común izquierda y derecha. ${ }^{12}$ Se midió con el paciente en decúbito supino en reposo y se efectuó un escaneo longitudinal de la arteria carótida común derecha e izquierda con ultrasonido modo B (Philips iE33 Apparatus with Transducer L11-3, $囚$ Yorba Linda, CA) a lo largo de un centímetro. Se realizaron mediciones en tres puntos y se obtuvo el valor promedio para obtener la medición del GIM. ${ }^{13-15}$ Se consideró anormal un GIM carotídeo $>0.37 \mathrm{~mm}$ en niños y niñas hasta los 6 años, $>0.41 \mathrm{~mm}$ en niños de 6 a 17 años 11 meses y $>0.39 \mathrm{~mm}$ en niñas de 6 a 17 años 11 meses de edad..$^{16}$

El riesgo cardiovascular se determinó de forma indirecta mediante los niveles séricos de la PCRus. ${ }^{17-19}$ Una muestra de $10 \mathrm{~mL}$ de sangre venosa periférica obtenida en ayuno de 12 horas se procesó mediante nefelometría. Se definió que el individuo tenía bajo riesgo cardiovascular si sus niveles eran $<1 \mathrm{mg} / \mathrm{L}$, con riesgo moderado $1-3 \mathrm{mg} / \mathrm{L}$ y con alto riesgo $>3 \mathrm{mg} / \mathrm{L}$. Se excluyeron los pacientes con valores $>10 \mathrm{mg} / \mathrm{L}$, por asociarse a procesos inflamatorios agudos. ${ }^{7}$

Para comparar las proporciones se empleó prueba de chi cuadrado o prueba exacta de Fisher. Para comparar variables continuas con distribución normal se empleó t Student. Como medida de asociación se obtuvo la razón de momios (RM) con intervalo de confianza de 95 \% (IC 95 \%). Se consideró como diferencia estadísticamente significativa una $p<0.05$.

\section{Resultados}

La edad promedio fue de $11.5 \pm 3$ años (rango 4 a 17). La AN fue leve en 16 sujetos (26.6\%), moderada en $13(21.6 \%)$ y severa en 31 (51.6\%). Se diagnosticó SM en 26 sujetos (43\%), sin diferencia entre sexos: 11 varones (42\%) versus 15 mujeres (58\%, $\mathrm{p}=0.58)$. La frecuencia de los componentes del SM fue HDL bajo en 26 sujetos (100\%), obesidad en 25 (96\%), triglicéridos elevados en 24 (92\%), hipertensión arterial en 10 (38\%) e hiperglucemia en uno (4\%).

La PCRus tuvo niveles promedio de $3.07 \pm 2.03 \mathrm{mg} / \mathrm{L}$ (rango 1 a 9.5). Todos los sujetos presentaron PCRus alterada: $40(67 \%)$ nivel de riesgo cardiovascular moderado y $16(27 \%)$ nivel de riesgo cardiovascular alto; cuatro sujetos (6\%) fueron eliminados por presentar niveles de PCRus $>10 \mathrm{mg} / \mathrm{L}$. De los 56 sujetos con PCRus alterada, hubo igual número por sexo: $28(50 \%, p=1.0)$.

El GIM promedio de la carótida común derecha fue de $0.44 \pm 0.04 \mathrm{~mm}$ (rango $0.40 \mathrm{a} 0.65 \mathrm{~mm}$ ) y de la carótida común izquierda de $0.44 \pm 0.03 \mathrm{~mm}$ (rango 0.40 a $0.55 \mathrm{~mm}$ ). Se encontraron valores del GIM carotídeo anormales para edad y sexo en 59 pacientes $(98 \%)$. De ellos, 29 (49\%) fueron masculinos y $30(51 \%)$ femeninos $(p=0.45)$.

La severidad de la AN no influyó en las variables investigadas (Tabla 1). Entre los niños con SM, las cifras promedio de presión arterial fueron estadísticamente mayores en el sexo masculino (Tabla 2). Los niveles elevados de triglicéridos y disminuidos de HDL fueron las alteraciones que confirieron a los niños con AN mayor posibilidad de desarrollar SM (Tabla 3).

\section{Discusión}

La frecuencia de SM en los niños y adolescentes mexicanos de nuestro estudio fue de $43 \%$, menor que la reportada en niños brasileños con AN (67.3\%), ${ }^{20}$ pero ambas superiores a las que se encuentran en población pediátrica abierta. Por ejemplo, en población mexicoamericana el SM se reportó en $18.7 \%{ }^{21}$ y 
Tabla 2. Variables de estudio por sexo en niños y adolescentes con síndrome metabólico

\begin{tabular}{|c|c|c|c|c|c|}
\hline \multirow[t]{2}{*}{ Componente } & \multicolumn{2}{|c|}{ Masculino $(n=11)$} & \multicolumn{2}{|c|}{ Femenino $(n=15)$} & \multirow[t]{2}{*}{$\mathbf{p}^{*}$} \\
\hline & Media $\pm \mathrm{DE}$ & Mín.-Máx. & Media \pm DE & Mín.-Máx. & \\
\hline Circunferencia cintura $(\mathrm{cm})$ & $102 \pm 12$ & $85-124$ & $96 \pm 14$ & $64-128$ & 0.28 \\
\hline PA sistólica (mmHg) & $117 \pm 15$ & $92-148$ & $103 \pm 16$ & $70-132$ & 0.03 \\
\hline PA diastólica (mmHg) & $69 \pm 10$ & $58-88$ & $60 \pm 10$ & $40-78$ & 0.04 \\
\hline Triglicéridos (mg/dL) & $171 \pm 42$ & $114-256$ & $152 \pm 53$ & $65-267$ & 0.34 \\
\hline Colesterol HDL (mg/dL) & $34 \pm 5$ & $25-40$ & $32 \pm 5$ & $19-39$ & 0.36 \\
\hline Glucosa (mg/dL) & $88 \pm 5$ & $79-94$ & $89 \pm 8$ & $76-111$ & 0.73 \\
\hline GIM carótida derecha (mm) & $0.44 \pm 0.03$ & $0.40-0.51$ & $0.43 \pm 0.02$ & $0.40-0.48$ & 0.76 \\
\hline GIM carótida izquierda (mm) & $0.43 \pm 0.03$ & $0.40-0.52$ & $0.44 \pm 0.03$ & $0.40-0.53$ & 0.53 \\
\hline PCRus (mg/L) & $4.03 \pm 2.86$ & $1.5-9.5$ & $2.85 \pm 1.59$ & $1-7.2$ & 0.31 \\
\hline
\end{tabular}

$\mathrm{PA}=$ Presión arterial, HOMA = Homeostasis Model Assessment, GIM = Grosor íntima-media, PCRus = Proteína C reactiva ultrasensible

Tabla 3. Distribución de variables en presencia de síndrome metabólico

\begin{tabular}{|c|c|c|c|c|c|c|c|}
\hline \multirow[t]{2}{*}{ Componente } & \multicolumn{2}{|c|}{ Con SM $(n=26)$} & \multicolumn{2}{|c|}{$\operatorname{Sin} \operatorname{SM}(n=34)$} & \multirow[t]{2}{*}{$\mathrm{p}$} & \multirow[t]{2}{*}{ RM } & \multirow[t]{2}{*}{ IC $95 \%$} \\
\hline & $\mathrm{n}$ & $\%$ & $\mathrm{n}$ & $\%$ & & & \\
\hline Sexo masculino & 11 & 42 & 19 & 56 & 0.58 & - & \\
\hline Sexo femenino & 15 & 58 & 15 & 44 & 0.58 & - & \\
\hline AN leve & 7 & 27 & 9 & 26 & 0.79 & - & \\
\hline AN moderada & 7 & 27 & 6 & 18 & 0.36 & - & \\
\hline AN severa & 12 & 46 & 19 & 56 & 0.58 & - & \\
\hline Circunferencia cintura $\geq 90$ & 25 & 96 & 25 & 74 & $0.03^{*}$ & 9 & $1-205$ \\
\hline Hipertensión arterial $\geq 90$ & 10 & 38 & 2 & 6 & $0.001^{* *}$ & 19 & $2-429$ \\
\hline Triglicéridos $\geq 110 \mathrm{mg} / \mathrm{dL}$ & 24 & 92 & 14 & 41 & $0.00004^{* *}$ & 30 & $3-660$ \\
\hline Colesterol HDL $\leq 40$ mg/dL & 26 & 100 & 14 & 41 & $0.00003^{* *}$ & $>30^{* * *}$ & \\
\hline Glucosa $\geq 110 \mathrm{mg} / \mathrm{dL}$ & 1 & 4 & 0 & 0 & 0.42 & - & \\
\hline Resistencia a la insulina & 18 & 69 & 15 & 44 & $0.05^{\star *}$ & 3 & $0.86-10$ \\
\hline Aterosclerosis carotídea & 25 & 96 & 34 & 100 & $0.04^{\star \star}$ & 3 & $0.89-11$ \\
\hline Riesgo cardiovascular moderado & 14 & 54 & 26 & 76 & 0.10 & - & \\
\hline Riesgo cardiovascular alto & 8 & 31 & 8 & 24 & 0.49 & - & \\
\hline
\end{tabular}

*Prueba exacta de Fisher dos colas, ${ }^{* *}$ Prueba de chi cuadrado, ${ }^{* \star *}$ Inexacta, $\mathrm{SM}=$ Síndrome metabólico, AN = acantosis nigricans, $\mathrm{HDL}=$ lipoproteínas de alta densidad.

en población mexicana en $19.6 \%,{ }^{22}$ resultados esperados si consideramos que en la patogénesis del SM están involucradas principalmente la obesidad y la resistencia a la insulina, ${ }^{23}$ presentes en niños con AN.

La importancia de esta frecuencia de SM es que los individuos que lo padecen en la infancia tienen en la etapa adulta un riesgo 2.7 a 3.4 mayor de presentarlo, dos veces más riesgo de desarrollar aterosclerosis carotídea y dos a tres veces más riesgo de desarrollar diabetes, ${ }^{24}$ además de un riesgo significativamente mayor de eventos cardiovasculares, con una prevalencia de $19.4 \%$, mientras que en aquellos sin SM es de $1.5 \% .{ }^{25}$

Los componentes más frecuentes del SM fueron niveles bajos de HDL, obesidad y niveles elevados de triglicéridos, sin diferencia significativa entre sexos. Las cifras de presión arterial sistólica y de presión arterial diastólica fueron estadísticamente más altas 
en el sexo masculino. Este último hallazgo es semejante al reportado por Halley et al., ${ }^{22}$ quienes observaron una frecuencia de hipertensión arterial significativamente mayor en mexicanos del sexo masculino, pero no en el resto de sus componentes.

La obesidad, los niveles elevados de triglicéridos y los niveles disminuidos de HDL son factores que aumentan el riesgo de SM en la edad adulta. De acuerdo con el Estudio del Corazón de Bogalusa, la obesidad confiere un riesgo 11 veces mayor para la aparición de este síndrome en la edad adulta. ${ }^{26}$ Según el Estudio Finlandés sobre Riesgo Cardiovascular en Jóvenes, los triglicéridos elevados, el colesterol HDL bajo y las cifras elevadas de tensión arterial son predictores de SM. ${ }^{27}$ En nuestro estudio, estos componentes confirieron mayor posibilidad en los niños y adolescentes con AN para el desarrollo de SM, en algunos casos hasta 30 veces más riesgo.

En cuanto a la PCRus, la evidencia acumulada sugiere que su medición representa un predictor de riesgo cardiovascular incluso más potente que los valores de LDL, tanto en pacientes con enfermedad coronaria como en sujetos aparentemente sanos. ${ }^{28} \mathrm{En}$ nuestro estudio todos los niños y adolescentes con AN presentaron niveles de PCRus considerados de riesgo moderado $O$ alto. No observamos diferencia de los niveles de PCRus entre niños con y sin SM, pero en un estudio que incluyó niños españoles los autores observaron que aquellos con obesidad y SM tuvieron cifras más elevadas de PCRus que aquellos con obesidad sin SM, por lo que la prueba se considera de utilidad para la detección temprana de factores de riesgo cardiovascular en este grupo etario. ${ }^{29}$

Respecto a la aterosclerosis, el proceso se inicia desde la infancia, mucho antes de que se presenten signos y síntomas identificables clínicamente. En autopsias realizadas en jóvenes se demostró que la aterosclerosis en estadios tempranos tuvo relación con obesidad, niveles elevados de triglicéridos y disminuidos de colesterol HDL. ${ }^{30}$ Estas alteraciones metabólicas estuvieron presentes en los niños y adolescentes incluidos en nuestro estudio, lo que podría explicar que $98 \%$ hubiera presentado aterosclerosis carotídea subclínica. No encontramos análisis que evaluaran el GIM carotídeo en niños con AN, pero su determinación es importante porque es un predictor de riesgo para enfermedad cardiovascular y cerebrovascular y por su correlación positiva con factores de riesgo, tanto clásicos como emergentes. ${ }^{23,30-32}$

Estas alteraciones en los niños y adolescentes mexicanos con AN tienen un impacto sobre la economía, pues en México la muerte prematura por sobrepeso y obesidad en 2008 implicó una pérdida de productividad por $\$ 1931$ millones de dólares. ${ }^{33}$ Por ello, la implementación de acciones efectivas de prevención durante la infancia debe ser una prioridad de los sistemas de salud, pues los programas de prevención podrían evitar entre 47000 y 55000 muertes por enfermedades crónicas por año. ${ }^{34}$

Aunque se requieren estudios longitudinales con mayor número de pacientes para corroborar los resultados, la exploración sistemática de niños y adolescentes en busca de AN podría ayudar a la detección oportuna de alteraciones metabólicas y cardiovasculares, para implementar medidas que prevengan la morbimortalidad en la vida adulta.

\section{Bibliografía}

1. Nguyen TT, Keil MF, Russell DL, Pathomvanich A, Uwaifo GI, Sebring NG, et al. Relation of acanthosis nigricans to hyperinsulinemia and insulin sensitivity in overweight African American and white children. J Pediatr. 2001;138:474-480.

2. Juárez-López $C$, Klünder-Klünder M, Medina-Bravo $P$, Madrigal-Azcárate A, Mass-Díaz E, Flores-Huerta S. Insulin resistance and its association with the components of the metabolic syndrome among obese children and adolescents. BMC Public Health. 2010;10:318.

3. Sinha S, Schwartz RA. Juvenile acanthosis nigricans. J Am Acad Dermatol. 2007;57:502-508.

4. Stoddart ML, Blevins KS, Lee ET, Wang W, Blackett PR, Cherokee Diabetes Study. Association of acanthosis nigricans with hyperinsulinemia compared with other selected risk factors for type 2 diabetes in Cherokee Indians: the Cherokee Diabetes Study. Diabetes Care. 2002;25:1009-1014.

5. Razani B, Chakravarthy MV, Semenkovich CF. Insulin resistance and atherosclerosis. Endocrinol Metab Clin North Am. 2008;37:603-621.

6. Organisation for Economic Co-operation and Development. [Sitio web]. Obesity Update 2014. [Consultado 2017 Jul 25]. Disponible en: http:// www.oecd.org/els/health-systems/Obesity-Update-2014.pdf

7. Pearson TA, Mensah GA, Alexander RW, Anderson JL, Cannon RO, Criqui $\mathrm{M}$, et al. Markers of inflammation and cardiovascular disease: application to clinical and public health practice. A statement for health care professionals from the Centers for Disease Control and Prevention and the American Heart Association. Circulation. 2003;107:499-511.

8. Burke JP, Hale DE, Hazuda HP, Stern MP. A quantitative scale of acanthosis nigricans. Diabetes Care. 1999;22:1655-1659.

9. Cook S, Weitzman M, Auinger P, Nguyen M, Dietz WH. Prevalence of a metabolic syndrome phenotype in adolescents: findings from the third National Health and Nutrition Examination Survey, 1988-1994. Arch Pediatr Adolesc Med. 2003;157:821-827.

10. Fernández JR, Reeden DT, Petrobielli A, Allison DB. Waist circumference percentiles in nationally representative sample of African-American, European-American and Mexican-American children and adolescent. J Pediatr. 2004;145:439-444.

11. National High Blood Pressure Education Program Working Group on High Blood Pressure in Children and Adolescents. The fourth report on the diagnosis, evaluation, and treatment of high blood pressure in children and adolescents. Pediatrics. 2004:114:555-576.

12. Amato M, Montorsi $P$, Ravani $A$, Oldani $E$, Galli $S$, Ravagnani $P M$, et al. Carotid intima-media thickness by B-mode ultrasound as surrogate of coronary atherosclerosis: correlation with quantitative coronary angiography and coronary intravascular ultrasound findings. Eur Heart J. 2007;28:2094-2101.

13. Pignoli P, Tremoli E, Poli A, Oreste P, Paoletti R. Intimal plus medial thickness of the arterial wall: a direct measurement with ultrasound imaging. Circulation. 1986;74:1399-1406.

14. Salonen R, Salonen JT. Determinants of carotid intima-media thickness: a population-based ultrasonography study in eastern Finnish men. J Intern Med. 1991;229:225-231.

15. Salonen JT, Salonen R. Ultrasound B-mode imaging in observational studies of atherosclerotic progression. Circulation. 1993;87:1156-1165. 
16. Doyon A, Kracht D, Bayazit AK, Deveci M, Duzova A, Krmar RT, et al. Carotid artery intima-media thickness and distensibility in children and adolescents: reference values and role of body dimensions. Hypertension. 2013;62:550-556.

17. Wang TJ, Gona P, Larson MG, Tofler GH, Levy D, Newton-Cheh C, et al. Multiple biomarkers for the prediction of first major cardiovascular events and death. N Engl J Med. 2006;355:2631-2639.

18. Asher J, Houston M. Statins and C-reactive protein levels. J Clin Hypertens (Greenwich). 2007;9:622-628.

19. DeBoer MD, Gurka MJ, Sumner AE. Diagnosis of the metabolic syndrome is associated with disproportionately high levels of high-sensitivity C-reactive protein in Non-Hispanic black adolescents: an analysis of NHANES 1999-2008. Diabetes Care. 2011;34:734-740.

20. Kluczynik CE, Mariz LS, Souza LC, Solano GB, Albuquerque FC, Medeiros CC. Acanthosis nigricans and insulin resistance in overweight children and adolescents. An Bras Dermatol. 2012;87:531-537.

21. Fowler SP, Puppala S, Arya R, Chittoor G, Farook VS, Schneider J, et al. Genetic epidemiology of cardiometabolic risk factors and their clustering patterns in Mexican American children and adolescents: the SAFARI Study. Hum Genet. 2013;132:1059-1071.

22. Halley-Castillo E, Borges G, Talavera JO, Orozco R, Vargas-Alemán C, Huitrón-Bravo $\mathrm{G}$, et al. Body mass index and the prevalence of metabolic syndrome among children and adolescents in two Mexican populations. J Adolesc Health. 2007;40:521-526.

23. García-García E, De-La-Llata-Romero M, Kaufer-Horwitz M, Tusié-Luna MT Calzada-León R, Vázquez-Velázquez V, et al. La obesidad y el síndrome metabólico como problema de salud pública. Una reflexión. Salud Publica Mex. 2008;50:530-547.

24. Magnussen CG, Koskinen J, Chen W, Thomson R, Schmidt MD, Srinivasan SR, et al. Pediatric metabolic syndrome predicts adulthood metabolic syndrome, subclinical atherosclerosis, and type 2 diabetes mellitus but is no better than body mass index alone: the Bogalusa Heart Study and the Cardiovascular Risk in Young Finns Study. Circulation. 2010;122:1604-1611.
25. Morrison JA, Friedman LA, Gray-McGuire C. Metabolic syndrome in childhood predicts adult cardiovascular disease 25 years later: the Princeton Lipid Research Clinics Follow-up Study. Pediatrics. 2007;120:340-345.

26. Srinivasan SR, Myers L, Berenson GS. Predictability of childhood adiposity and insulin for developing insulin resistance syndrome (syndrome $\mathrm{X}$ ) in young adulthood: the Bogalusa Heart Study. Diabetes. 2002;51:204-209.

27. Raitakari OT, Porkka KV, Rönnemaa T, Knip M, Uhari M, Akerblom HK, et al. The role of insulin in clustering of serum lipids and blood pressure in children and adolescents. Diabetologia. 1995;38:1042-1050.

28. Arroyo-Espliguero R, Avanzas P, Kaski JC. Enfermedad cardiovascular ateroesclerótica: la utilidad de la proteína $C$ reactiva en la identificación de la placa vulnerable y del paciente "vulnerable". Rev Esp Cardiol. 2004;57:375-378.

29. Soriano-Guillén L, Hernández-García B, Pita J, Domínguez-Garrido N, Del-Río-Camacho G, Rovira A. High-sensitivity C-reactive protein is a good marker of cardiovascular risk in obese children and adolescents. Eur J Endocrinol. 2008;159:1-4.

30. Berenson GS, Srinivasan SR, Bao W, Newman HP, Tracy RE, Wattigney WA. Association between multiple cardiovascular risk factors and atherosclerosis in children and young adults. The Bogalusa Heart Study. N Engl J Med. 1998;338:1650-1656.

31. McGill HC, McMahan CA, Herderick EE, Zieske AW, Malcom GT, Tracy RE, et al. Obesity accelerates the progression of coronary atherosclerosis in young men. Circulation. 2002;105:2712-2718.

32. Bots ML, Evans GW, Riley WA, Grobbee DE. Carotid intima-media thickness measurements in intervention studies: design options, progression rates, and sample size considerations: a point of view. Stroke. 2003;34:2985-2994.

33. Rtveladze K, Marsh T, Barquera S, Sanchez-Romero LM, Levy D, Melendez $\mathrm{G}$, et al. Obesity prevalence in Mexico: impact on health an economic burden. Public Health Nutr. 2014;17:233-239.

34. Obesity and the economics of prevention: Fit not fat. Key facts-Mexico, update 2014. France: Organization for Economic Cooperation and Development; 2014. 